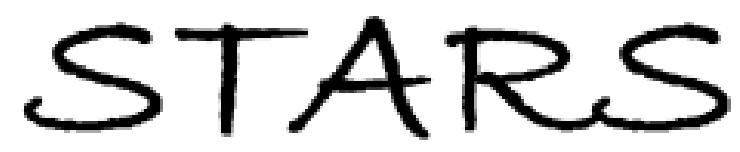

University of Central Florida

STARS

$1-1-2003$

\title{
Dependence of two-photon-absorption-excited fluorescence on the angle between the linear polarizations of two intersecting beams
}

\author{
Alexandra Rapaport \\ University of Central Florida \\ Ferenc Szipocs \\ University of Central Florida \\ Michael Bass \\ University of Central Florida
}

Find similar works at: https://stars.library.ucf.edu/facultybib2000

University of Central Florida Libraries http://library.ucf.edu

This Article is brought to you for free and open access by the Faculty Bibliography at STARS. It has been accepted for inclusion in Faculty Bibliography 2000s by an authorized administrator of STARS. For more information, please contact STARS@ucf.edu.

\section{Recommended Citation}

Rapaport, Alexandra; Szipocs, Ferenc; and Bass, Michael, "Dependence of two-photon-absorption-excited fluorescence on the angle between the linear polarizations of two intersecting beams" (2003). Faculty Bibliography 2000s. 3980.

https://stars.library.ucf.edu/facultybib2000/3980

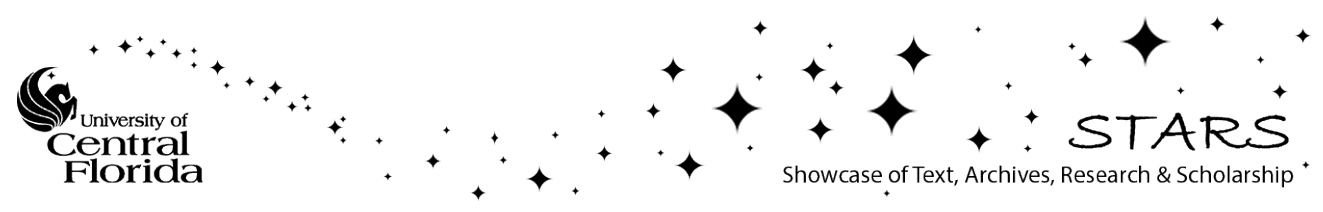




\section{Dependence of two-photon-absorption- excited fluorescence on the angle between the linear polarizations of two intersecting beams}

Cite as: Appl. Phys. Lett. 82, 4642 (2003); https://doi.org/10.1063/1.1585131

Submitted: 03 February 2003 . Accepted: 23 April 2003. Published Online: 24 June 2003

Alexandra Rapaport, Ferenc Szipöcs, and Michael Bass

ARTICLES YOU MAY BE INTERESTED IN

Low-loss, high-bandwidth graded-index plastic optical fiber fabricated by the centrifugal deposition method

Applied Physics Letters 82, 4645 (2003); https://doi.org/10.1063/1.1584516

Polarization Dependence of the Two-Photon Absorption of Tumbling Molecules with Application to Liquid 1-Chloronaphthalene and Benzene

The Journal of Chemical Physics 53, 29 (1970); https://doi.org/10.1063/1.1673778

Applied Physics Letters

Mid-IR and THz frequency combs special collection

Read Now! 


\title{
Dependence of two-photon-absorption-excited fluorescence on the angle between the linear polarizations of two intersecting beams
}

\author{
Alexandra Rapaport, Ferenc Szipöcs, and Michael Bass ${ }^{\mathrm{a})}$ \\ School of Optics/CREOL, University of Central Florida, Orlando, Florida 32816
}

(Received 3 February 2003; accepted 23 April 2003)

\begin{abstract}
We report experimental observation of the dependence of the fluorescence excited by two-photon absorption (TPA/E fluorescence) in dye solutions on the angle between the linear polarizations of two intersecting pump beams. Both degenerate and nondegenerate TPA/E fluorescence were studied with $4 \mathrm{~ns}$ pulsed tunable lasers as pump sources. We find that earlier theoretical analyses do not contain all of the observed changes in fluorescence signal with relative polarization angle. (C) 2003 American Institute of Physics. [DOI: 10.1063/1.1585131]
\end{abstract}

We report experimental studies of the fluorescence excited by two-photon absorption (TPA/E fluorescence) in the intersection of two linearly polarized beams in which attention is given to the effect of the relative orientation of the polarization directions of the beams. Both degenerate (e.g., when both beams are at the same wavelength) and nondegenerate (e.g., when each beam is at a different wavelength) TPA/E fluorescence were studied. Our experimental observations of the polarization dependence effect for several dyes, with rhodamine B in methanol as our exemplar, indicate that suggested models for this phenomenon are incomplete. ${ }^{1-7}$

The experiment is shown schematically in Fig. 1. The sources were two optical parametric oscillators, each in a master oscillator-power oscillator (MOPO) configuration, which could be independently tuned from $\sim 440$ to $690 \mathrm{~nm}$ in the signal and $\sim 725$ to $1900 \mathrm{~nm}$ in the idler outputs. They were pumped by the third harmonic of a $10 \mathrm{~Hz}$, flashlamp pumped, $Q$-switched Nd:yttritium-aluminum-garnet laser. The MOPO output pulses were about $4 \mathrm{~ns}$ long and at the sample did not exceed $4 \mathrm{~mJ}$ at any wavelength. They arrived within $0.5 \mathrm{~ns}$ of each other at their intersection where each beam radius was about $500 \mu \mathrm{m}$.

The Berek polarization compensator (BPC) was adjusted $^{8,9}$ to allow us to rotate the polarization of beam 1 in Fig. 1 from parallel to perpendicular to that of beam 2 . The rotation angle is designated as $\theta$ and $\theta=0^{\circ}$ indicates parallel polarizations. We checked the properties of the BPC experimentally. Its attenuation of the beam was independent of the selected output linear polarization. Adjusting the orientation of the BPC did not cause noticeable displacement of the transmitted beam. This assured that the effects reported are not due to changes in the volume of the beams' intersection.

The visible emission due to the two intersecting beams was observed in a direction perpendicular to their plane of propagation as sketched in Fig. 1. This light was collected with a short-focal-length lens and passed through an aperture that was imaged onto either a charge coupled device (CCD) camera or the entrance slit of a monochromator. The CCD camera allowed visualization of the intersection of the two beams and adjustment of their overlap for maximum emis-

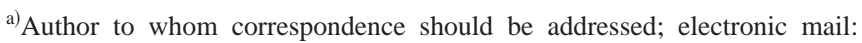
mbass@mail.ucf.edu
}

sion. The aperture was then centered on the light coming from the beams' intersection and closed down so that only light from the region of the intersection was collected. The fluorescence excited by two-photon absorption at the intersection of the two beams then passed through a monochromator and was detected by a photomultiplier-oscilloscope combination. We also monitored the energy of both exciting beams.

Figure 1 shows that the geometry of the two intersecting beams forced us to detect the visible emission along an optical axis parallel to the polarizations of the beams when $\theta$ $=0^{\circ}$. If the TPA induced dipole moment of the excited molecules for visible emission were along this direction then the signal detected for $\theta=0^{\circ}$ would be minimal. However, because we worked with $\sim 4$ ns pulses the dye molecules could reorient during excitation to emit light that we could detect. This consideration led us to examine the role of solvent viscosity on the effects we observed. For high viscosity solvents such as cyclohexanol, the maximum to minimum fluorescence signal ratio when $\theta$ was varied from $0^{\circ}$ to $90^{\circ}$ was less than observed with low viscosity solvents for the same dye.

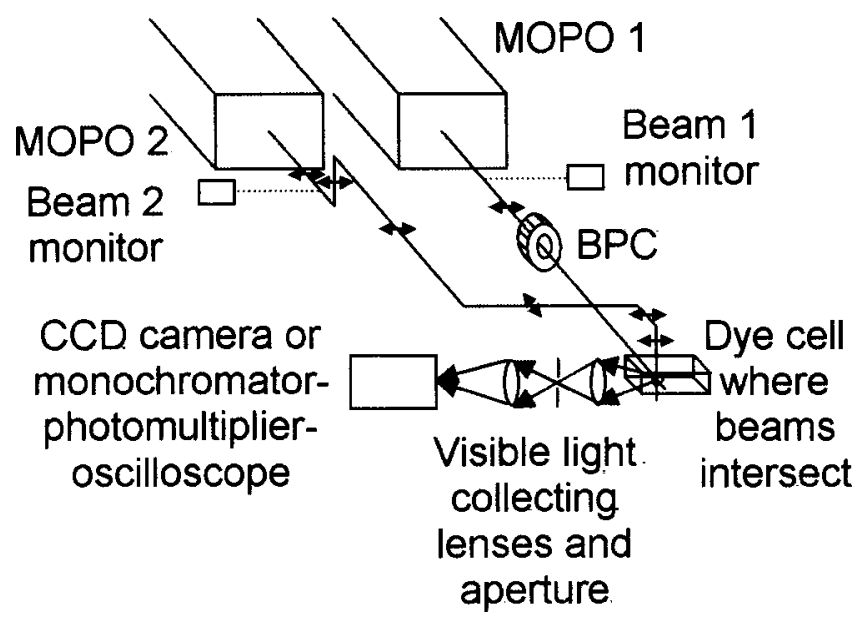

FIG. 1. Sketch of the experiment in which the beam paths have been simplified for clarity. $45^{\circ}-45^{\circ}-90^{\circ}$ prisms were used to turn the beams except where the beams were split to monitor the energy in each beam. Double headed arrows show the direction of the polarization in each leg of the beam path. The polarization angle of beam 1 can be set by the BPC to change the angle $\theta$ with respect to the polarization of beam 2 . Each beam was focused with a $20 \mathrm{~cm}$ focal length lens to a diameter at the intersection of $\sim 0.5 \mathrm{~mm}$. 


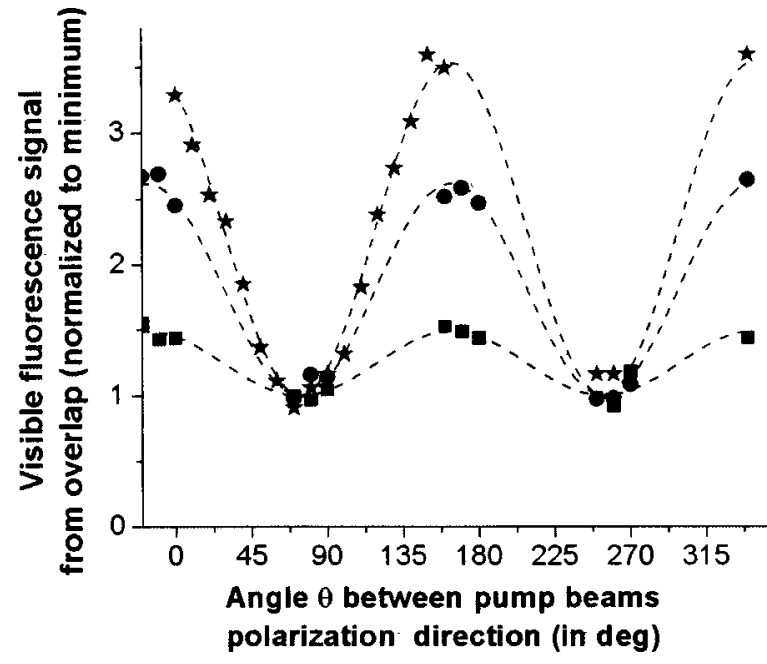

FIG. 2. Nondegenerate TPA/E fluorescence from rhodamine B in methanol at $590 \mathrm{~nm}$ normalized to unity at the minimum vs the angle $\theta$ between the polarizations of the pump beams. The excitation wavelengths were: $(\star) 750$ and $965 \mathrm{~nm}$, (○) 920 and $1200 \mathrm{~nm}$, and (ם) 920 and $1367 \mathrm{~nm}$. The symbols are experimental data points and the dashed curves are plots of the function $1+A \cos ^{2} \theta$ where $A$ is chosen to fit each data set.

In high viscosity solvents the reorientation required for detection is minimized and a lower maximum signal is detected. We note here that the assumption of the orientation of the emitting dipoles is just that and the explanation of the viscosity dependence may be more complex. Molecular reorientation may contribute to some of the anomalies mentioned later and should be studied further.

The visible emission detected for each excitation beam was recorded separately and summed to serve as a measure of the total visible emission due to each beam alone without interacting with each other. When both beams were simultaneously present we obtained a measure of the nondegenerate two-photon excitation process only by subtracting the total signal from each beam alone without interaction.

Coumarin 7 is a dye of interest to our studies of scalable media for three-dimensional (3D) displays. ${ }^{10}$ It has a single TPA spectral feature centered at $880 \mathrm{~nm}$ with a full width at half maximum of $\sim 150 \mathrm{~nm}$. When excited by beams at 730 and $1050 \mathrm{~nm}$ nondegenerate TPA/E fluorescence is observed. It shows the expected dependence on the product of the energies in the beams, e.g., $E_{1} E_{2}$, and a dependence on the angle $\theta$ given by $1+2 \cos ^{2} \theta$ as predicted by existing models for the relative polarization effect. ${ }^{1,2}$ In the following we present evidence that other dyes do not behave as these models predict.

The TPA excitation spectrum of rhodamine B in methanol presents several features as reported in the past ${ }^{11,12}$ and confirmed by our own experiments: that is TPA peaks around 550, 517, and $422 \mathrm{~nm}$. Pairs of excitation wavelengths were selected such that each beam did not produce significant TPA/E fluorescence by itself but the interaction of the two beams at their intersection produced a strong signal. In Fig. 2 , we show the experimentally observed dependence of TPA/E fluorescence on $\theta$ for three pairs of excitation wavelengths that accessed each of the TPA peaks: 750 and $965 \mathrm{~nm}$ for the peak at $422 \mathrm{~nm}, 920$ and $1200 \mathrm{~nm}$ for the peak at 517 $\mathrm{nm}$, and 920 and $1367 \mathrm{~nm}$ for the peak at $550 \mathrm{~nm}$. The data in Fig. 2 were obtained when the energies in the two pump

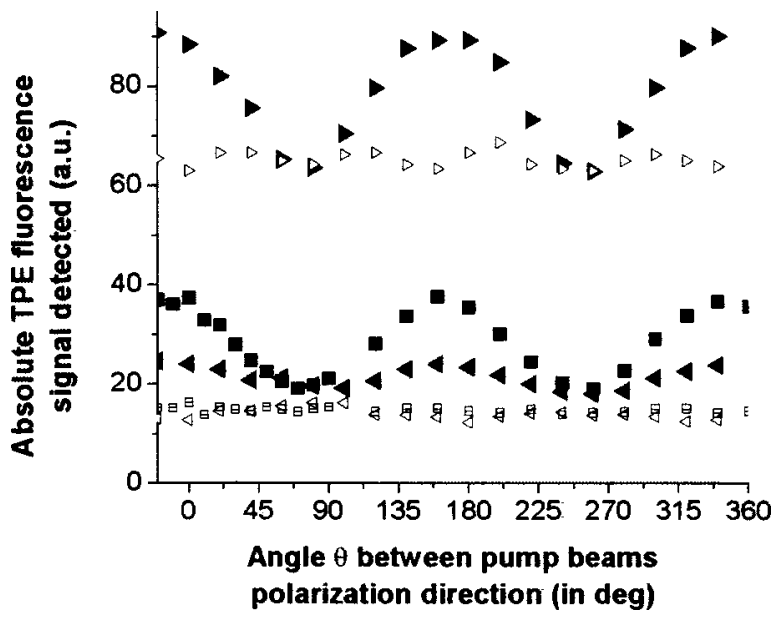

FIG. 3. Nondegenerate TPA/E fluorescence from rhodamine B in methanol at $590 \mathrm{~nm}$ vs the angle $\theta$ for pump beams at 750 and $965 \mathrm{~nm}$ for three sets of energy in the beams chosen in a way that the product of the energies is constant. The ( ) is data from case I where there is ten times more energy in the $965 \mathrm{~nm}$ pump beam, the ( $\mathbf{\square})$ is data from case II with equal energy in both beams and the $(4)$ is data from case III with ten times more energy in the $750 \mathrm{~nm}$ beam. The solid symbols are measurements of the total fluorescence collected when the two beams overlap, and the hollow symbols are the sum of the fluorescence measured when each beam passes through the sample individually.

beams were nearly equal. In this figure the dashed curves are fits of the data to the functional form $1+A \cos ^{2} \theta$ with $A$ adjusted for each data set. Clearly shown in Fig. 2 is the fact that the magnitude of the polarization dependence is determined by the excited state reached. ${ }^{1,2,13}$ Also, the ratio of maximum to minimum TPA/E fluorescence is greater than 3 in the case of the higher lying excited state. This observation runs counter to the prediction of a simple geometrical model described in Ref. 14 based on averaging of the interaction of the optical fields with randomly oriented dye molecules.

The same experiment was repeated with several other fluorophores such as fluorescein and naphtofluorescein in methanol and the same trend was observed: for dyes having several TPA peaks, the shorter the wavelength of the peak accessed (e.g., the greater the energy of the state attained), the greater the ratio of maximum to minimum TPA/E fluorescence signal.

We investigated the dependence of the TPA/E fluorescence on the energy in the pump beams. Figure 3 presents plots of such fluorescence emitted from the intersection of the two pump beams measured for three conditions of pump energies such that the product of the energies was constant. (If the process were strictly nondegenerate TPA then the observed signal would depend linearly on the product of the two beams' energies as observed for coumarin 7.) In case I the beam at $965 \mathrm{~nm}$ is ten times more energetic than the beam at $750 \mathrm{~nm}$. In case II the energies are equal and in case III the $750 \mathrm{~nm}$ beam is ten times more energetic than the one at $965 \mathrm{~nm}$. The open symbols indicate the sum of the degenerate signals when the two pump beams are present separately while the solid symbols indicate the total signal detected when both beams are present simultaneously. In case I, the degenerate signal due to both beams alone (represented by the $\triangleright$ data) is quite strong, leading to a much brighter background signal than when the $750 \mathrm{~nm}$ beam carries more energy (represented by the $\triangleleft$ data). Also there appears to be 
very little nondegenerate TPA/E fluorescence for perpendicular polarization when the long-wavelength beam carries most of the energy [represented by the $(\boldsymbol{)})$ data], while the signal increases by about $40 \%$ when $\theta=0^{\circ}$. When the two beams have about the same energy as in case II [represented by the (ם) symbols], there is some nondegenerate TPA/E fluorescence for perpendicular polarization. However, the total visible emission still doubles when the relative polarization is changed from perpendicular to parallel polarization (the nondegenerate part alone more than triples). Finally, in case III there is very little variation in the visible fluorescence emitted when $\theta$ is varied through a full $360^{\circ}[(\varangle)$ symbols]. As a result, the ratio of maximum to minimum nondegenerate TPA/E fluorescence signal is very small in case III.

Most earlier studies of TPA/E fluorescence were performed with degenerate excitation and models were proposed to describe the process. Monson and $\mathrm{McClain}^{1}$ derived the expression $A+B \cos ^{2} \theta$ to describe the polarization effect as early as 1970. They also discussed the role of molecular wavefunction symmetry on the values of $A$ and $B$ in the process of degenerate TPA/E fluorescence. We presented a model giving the expression $1+2 \cos ^{2} \theta$ based on averaging the contribution to TPA/E from randomly oriented linear molecules. ${ }^{14}$ This model did not distinguish degenerate and nondegenerate TPA/E. Meath and Power ${ }^{7}$ also evaluate a polarization orientation dependence for both degenerate and nondegenerate TPA/E fluorescence without finding a dependence on power in each beam as we observe. Continued theoretical analyses are required and are in progress. For example, the use of nanosecond excitation in our case could enhance the effect of excited state absorption (ESA) of the $750 \mathrm{~nm}$ radiation. ${ }^{15-17}$ However, no ESA model yet examined was able to explain the apparent lack of interaction of the two pump beams when $\theta=90^{\circ}$ in case I. A further consequence of using nanosecond pulses could be an ordering of the molecules' orientation in solution. This would render inadequate any averaging over space that is performed in the existing models and might explain the discrepancies with our practical results.
In summary, we find experimentally that the polarization dependence of nondegenerate TPA/E fluorescence in rhodamine B in methanol and other dye solutions is more complex than considered in existing models. Since TPA excitation is essential to such applications as 3D displays, fluorescence microscopy, optical limiting, and optical memories the polarization dependence must be more thoroughly understood and taken into account.

The authors gratefully acknowledge the support of this work by the U.S. Army Research Office Grant No. DAAD19-99-1-0220 and DURIP Award No. DAAD19-0010132.

${ }^{1}$ P. R. Monson and W. M. McClain, J. Chem. Phys. 53, 29 (1970).

${ }^{2}$ W. M. McClain, J. Chem. Phys. 55, 2789 (1971).

${ }^{3}$ B. N. Jagatap, W. J. Meath, D. Tittelback-Helmrich, and R. P. Steer, J. Chem. Phys. 103, 121-135 (1995).

${ }^{4}$ B. N. Jagatap and W. J. Meath, Chem. Phys. Lett. 258, 293 (1996).

${ }^{5}$ P. Tran, W. J. Meath, B. D. Wagner, and R. P. Steer, J. Chem. Phys. 100, 4165 (1994)

${ }^{6}$ D. A. Akimov, A. B. Fedotov, N. I. Koroteev, S. A. Magnitskii, A. N. Naumov, D. A. Sidorov-Biryukov, and A. M. Zheltikov, Jpn. J. Appl. Phys., Part 1 36, 426 (1997).

${ }^{7}$ W. J. Meath and E. A. Power, J. Phys. B 17, 763 (1984).

${ }^{8}$ M. Berek, pp. 427, 464, 580 (1913) [quoted in M. Born and E. Wolf, Principles of Optics, 6th ed. (Pergamon, London, 1980), p. 694]. New Focus, Inc. model 5540.

${ }^{9}$ F. S. Luecke, (New Focus, Inc., USA, 1992).

${ }^{10}$ A. Rapaport, K. Ayrault, E. S. Matthew-Daniel, and M. Bass, Appl. Phys. Lett. 74, 329 (1999).

${ }^{11}$ C. Xu and W. W. Webb, J. Opt. Soc. Am. B 13, 481 (1996).

${ }^{12}$ T. Wakebe and E. van Keuren, Jpn. J. Appl. Phys., Part 1 38, 3556 (1999).

${ }^{13}$ M. Rasmusson, A. N. Tarnovsky, E. Akesson, and V. Sundstrom, Chem. Phys. Lett. 335, 201 (2001).

${ }^{14}$ A. Rapaport, F. Szipocs, and M. Bass, Technical Digest. Summaries of papers presented at the Quantum Electronics and Laser Science Conference. Conference ed. (IEEE Cat. No. 02CH37338), (2002), Vol. 1, p. 51.

${ }^{15}$ D. J. Bradley, M. H. R. Hutchinson, H. Koetser, T. Morrow, G. H. C. New, and M. S. Petty, Proc. R. Soc. London, Ser. A 328, 97 (1972).

${ }^{16}$ S. Venugopal Rao, N. K. M. Naga Srinivas, and D. Narayana Rao, Chem. Phys. Lett. 361, 439 (2002).

${ }^{17}$ A. Fischer, C. Cremer, and E. H. K. Stelzer, Appl. Opt. 34, 1989 (1995). 